present. Educational programmes with specific interventions are recommended to increase KAPs and to prevent new HIV infections among this population

\section{P3.48 CHARACTERIZACION OF THE GESTATIONAL OUTCOME OF INFANTS BORN TO HIV POSITIVE MOTHERS IN ANTIRETROVIRAL TREATMENT DURING PREGNANCY}

${ }^{1} \mathrm{JC} O \mathrm{C}$ Crispim, ${ }^{2} \mathrm{KR}$ Torres, ${ }^{3} \mathrm{GM}$ Palomino, ${ }^{4} \mathrm{PE}$ A Siqueira, ${ }^{1} \mathrm{MN}$ Monteiro, ${ }^{5} \mathrm{MT}$ A Lima, ${ }^{1}$ DA Pascoal. 'Maternity School Januário Cicco, Universidade Federal do Rio Grande do Norte, Natal - RN, Brazil; ${ }^{2}$ Medicine Department, Universidade Federal do Rio Grande do Norte, Natal - RN, Brazil; ${ }^{3}$ Postgraduate Program in Pharmaceutical Science, Universidade Federal do Rio Grande do Norte, Natal - Rn, Brazil; ${ }^{4}$ Pharmacy Department, Universidade Federal do Rio Grande do Norte, Natal - RN, Brazil; ${ }^{5}$ Hospital of Paediatrics Teacher Heriberto Ferreira Bezerra, Universidade Federal do Rio Grande do Norte, Natal - RN, Brazil

10.1136/sextrans-2017-053264.285

Introduction Antiretroviral regimens used for the prevention of mother-to-child transmission of the human immunodeficiency virus (HIV) have shown benefits. However, combination antiretroviral therapy (ART) for the mother and infant have shown higher rates of adverse pregnancy outcomes. The objective of this research is to characterise the gestational outcome of infants born to HIV-positive mothers receiving highly active antiretroviral therapy (HAART) in a tertiary maternity unit through the evaluation of the clinical, anthropometric and epidemiological profile of the babies at birth.

Methods A cross-sectional study was carried out on HIV positive pregnant women at the Januário Cicco Maternity School (MEJC), whose childbirths occurred in the period from January 2010 to December 2014, being included 82 mothers and 84 babies. The research will continue in the year 2017.

Results The group of mothers selected had a mean age of 29.5 years and had their children with a mean gestational age of 36.7 weeks. Of these, $3.6 \%$ received a triple therapeutic regimen with combination of nucleoside reverse transcriptase inhibitor (NRTI) +2 protease inhibitors (PI), 4.9\% received regimen with 2 NRTI +1 Non-nucleoside reverse transcriptase inhibitor and $91.5 \%$ received quadruple regimen with 2 NRTI +2 PI. According to the information collected in the present study, only $17.8 \%$ of the babies were born prematurely. As for anthropometry, 22.6\% were born with low weight; $28.6 \%$ had cephalic perimeter and $15.5 \%$ length below the lower limits considered normal; and $98.8 \%$ of the babies reached.

Conclusion This type of characterisation is fundamental in a scenario where changes in the neonatal characteristics observed in the study generate a cost for maternal and child health, involving economics and management aimed at a specific follow-up of HIV positive mothers and their children exposed to the virus and to HAART during gestation. For this reason, it is necessary the specialised and multiprofessional clinical follow-up for the binomial in question.

\section{P3.49 CONTRIBUTION TO THE STUDY OF EPIDEMIOLOGICAL SURVEILLANCE OF CONGENITAL SYPHILIS IN A HOSPITAL OF THE PUBLIC HEALTH SYSTEM IN RJ, BRAZIL, JANUARY 2013 TO JANUARY 2017}

Cunha Aa, C Galvão, WC Borges, P Godefroy, Teixeira Sam, M Glinardello, E Gerde. Hospital Maternidade Therezinha de Jesus, Rio de Janeiero - RJ, Brazil

10.1136/sextrans-2017-053264.286
Introduction Syphilis consists of an STD (sexually transmitted disease) of bacterial aetiology, featuring a serious public health problem. The objective was to describe the prevalence of congenital syphilis in a hospital of the Public Health System Rio de Janeiro, Brazil.

Methods Design. A descriptive and observational research of quantitative and retrospective nature. Data collection. We collected data from the medical records. Statistical analysis. The data were processed by simple frequency measures (absolute and relative).

Results We analysed 175 notified CS (congenital syphilis) cases in 6274 deliveries (prevalence of 2,7\%). Some women at the time of delivery had their data collected, were affected by syphilis during pregnancy and prenatal care. About of $80.0 \%$ of women with syphilis performed prenatal care. There were carried out positive 276 tests (VDRL) in the Hospital Estadual da Mãe by the women in the study, 104 (59.42\%) in prenatal care and $172(98.28 \%)$ at time of delivery, when the test was repeated for all the participants. The distribution of the number of sexual partners of syphilitic pregnant women that made treatment during prenatal care of pregnant women was very impaired because of very high missing values.

Conclusions At the end of this study we found that even though the Ministry of Health has established a program together with the World Health Organisation, the goal of reducing the number of cases of congenital syphilis has not yet been reached. We have problems with the prenatal care, lack of effective screening program and monitoring of the pregnant woman. The findings of CS in the State Hospital of Mother separated for investigation were considered high in relation to the proposal drawn up by the Ministry of Health $(1 / 1,000)$.

\section{P3.50 SPECTRUM OF MALIGNANCIES AMONG PEOPLE LIVING WITH HIV (PLHIV) IN SOUTHERN INDIA}

D Madi, A Aliasgar, J Ramapuram. Department of Medicine, KMC Mangalore (Manipal University), India

\subsection{6/sextrans-2017-053264.287}

Introduction Non-Hodgkin's Lymphoma (NHL), Kaposi’s sarcoma and invasive carcinoma of cervix are AIDS defining cancers (ADCs) seen in people living with HIV (PLHIV). After the introduction of combination antiretroviral therapy (cART) the spectrum of malignancies has changed and the incidence of Non AIDS defining Cancers (NADCs) are increasing as PLHIV live longer.This study describes the pattern of malignancies among PLHIV attending a tertiary care HIV facility.

Methodology This descriptive cross sectional study was carried out at Kasturba Medical College (KMC), Mangalore which is a 500 bedded tertiary care referral institution. The study was conducted among PLHIV diagnosed with malignancy between January 2005 and May 2016. Data of 33 PLHIV was collected by using a semi-structured proforma after obtaining permission from the institutional ethics committee. Data was analysed by using SPSS version 11.5 statistical software.

Results The mean age of our study group was $46.19 \pm 7.82$ years. Majority of them $18(54.5 \%)$ were males. In the current study $19(57.6 \%)$ had Non AIDS defining cancers and 14 (42.4\%) had AIDS defining cancers. Non-Hodgkin's lymphoma 9 (27.2\%), carcinoma breast 5 (15.2\%), invasive carcinoma of cervix $5(15.2 \%)$ and carcinoma of head and neck 4 
(12.1\%) were the major malignancies seen in our study. Carcinoma lung 3 (9.1\%), Hodgkin's lymphoma 2 (6.1\%),carcinoma anal canal 2 (6.1\%) and 1 case each of Acute myeloid leukaemia, carcinoma colon and Ewing's sarcoma was seen in our study. The median $\mathrm{CD}_{4}$ count at the diagnosis of malignancy was 214 IQR(159-436) cells/ $\mu$ l. In our study 24 $(72.7 \%)$ patients were on cART at the time of diagnosis of the cancer.

Conclusion In our study the percentage of NADCs was more when compared to ADCs. Non- Hodgkin's lymphoma was the most common cancer seen in our study population. Kaposi sarcoma was not seen in our study population confirming the fact that it is a rare malignancy among PLHIV in our country. With longer survival of PLHIV in India due to potent cART malignancy will become an important issue for HIV physicians.

\section{P3.51 ABSTRACT WITHDRAWN}

\section{P3.52 AWARNESS OF HIVIAIDS AMONG MALAYALI TRIBES, YELAGIRI HILLS}

Delfin Lovelina Francis. Dr MGR Medical University, Chennai, India

\subsection{6/sextrans-2017-053264.288}

Introduction Despite remarkable world-wide progress in the field of diagnostic, curative and preventive medicine, still there are large populations of people living in isolation in natural and unpolluted surroundings far away from civilisation, maintaining their traditional values, customs, beliefs and myths. They are commonly known as tribes and are considered to be the indigenous people of the land. This study aimed to assess HIV-related knowledge, attitudes and practices among Malayali tribes, Yelagiri Hills, Tamil Nadu, India.

Methods A cross-sectional study on Malayali tribes aged between 20 and 30 years old was undertaken to evaluate their KAPs. We selected 200 eligible samples through systematic random sampling from different villages of Yelagiri Hills.

Results The majority of the population was unaware of HIV (49\%). Of the population who were aware 59\% knew that it can be transmitted by sexual intercourse and $88 \%$ from mother to child. Misconceptions about transmission of HIV were observed among $39.3 \%$ to $44.3 \%$ of respondents. More $75 \%$ mentioned village health workers as major sources of information on HIV/AIDS.

Conclusions Despite adequate knowledge about HIV/AIDS, misconceptions about routes of transmission were found. Negative attitudes to HIV/AIDS and risky practices were also present. Educational programmes with specific interventions are recommended to increase KAPs and to prevent new HIV infections among this population. It was recommended to increase KAPs and to prevent new HIV infections among this population.

\section{P3.53 LOST OPPORTUNITIES INVESTIGATION TO PREVENTION MOTHER-TO-CHILD TRANSMISSION HIV WITH LATE DIAGNOSIS}

Doris Sztutman Bergmann. 1 CCD/COVISA - SMS/Prefeitura Municipal de São Paulo, São Paulo - SP, Brazil

\subsection{6/sextrans-2017-053264.289}

In 2011, the city of São Paulo started investigations on lost opportunities for HIV mother-to-child transmission prevention on notified children born since 2000, infected by their mother's HIV. Main office team, responsible for STI/HIV epidemiological surveillance, controls the new mother-to-child HIV transmission notifications, sending each case to their home region. The local teams investigate the cases on diagnostic sites, following, notification and, if necessary, family or caregiver's interviews. Data collected on mothers and children are registered on a database, improved and analysed on Microsoft Excel. From 2011 to 2016, 188 children born between 2000 and 2015 which were infected by mother's HIV were investigated. From which, 44 mothers $(23,4 \%)$ were diagnosed before pregnancy, 25 (13,3\%) during pregnancy, $22(11,7 \%)$ during labour, 68 (32,2\%) after labour and $29(15,4 \%)$ there was no information. Among the 188 investigated mothers, $117(62,2 \%)$ attended to prenatal consults, $42(22,4 \%)$ didn't and there was no information on the other $29(15,4 \%) .13(13,4 \%)$ of the 97 women diagnosed after labour or without information did not attend to prenatal consults, 4 $(4,1 \%)$ did it late, $2(2,1 \%)$ denied to do it, on 2 of them $(2,1 \%)$ the exams were not prescribed and $21(21,6 \%)$ of them had negative result on the exam. Among those 97 women, 52 $(53,6 \%)$ attended to prenatal consults while 21 (21,6\%) didn't, and the 24 remaining $(24,8 \%)$ there's no data. From the 52 that attended to prenatal consults, but had after labour diagnostic or without this information, $46(88,5 \%)$ breastfed their children and $15(28,8 \%)$ didn't give their children the antiretroviral drugs properly. Whereas women may be infected at the end of pregnancy, making harder diagnostic or during breastfeeding, close follow-up of just delivered woman, with periodic HIV testing and the incentive to using condoms among breastfeeding and pregnant women is important. Moreover, women more vulnerable to HIV must be encouraged to do the tests, prenatal and postnatal.

\section{P3.54 ABSTRACT WITHDRAWN}

\section{P3.55 PATTERN OF SYPHILIS AND HIV CO-INFECTIONS AMONG ART TREATMENT NAÏVE ADULTS IN A TERTIARY INSTITUTION IN IBADAN, NIGERIA}

${ }^{1} S A$ Fayemiwo*, ${ }^{2} \mathrm{OA}$ Adesina, ${ }^{3} \mathrm{M}$ Obaro, ${ }^{2} \mathrm{O}$ Awolude, ${ }^{4} \mathrm{JO}$ Akinyemi, ${ }^{5} \mathrm{O}$ Mosuro, ${ }^{6} \mathrm{MO}$ Kuti, ${ }^{8} \mathrm{GN}$ Odaibo, ${ }^{2} \mathrm{FF}$ Adewole. ${ }^{1}$ Department of Medical Microbiology and Parasitology, College of Medicine, University of Ibadan, Ibadan, Nigeria; ${ }^{2}$ Department of Obstetrics and Gynaecology, College of Medicine, University of Ibadan, Ibadan; ${ }^{3}$ Department of Pharmacology and Therapeutics, College of Medicine, University of Ibadan, Ibadan, Nigeria; ${ }^{4}$ Department of Epidemiology and Medical Statistics, College of Medicine, University of Ibadan, Ibadan, Nigeria; ${ }^{5}$ Department of Family Medicine, University College hospital, Ibadan, Nigeria; ${ }^{6}$ Department of Chemical Pathology, College of Medicine, University of Ibadan, Ibadan, Nigeria; 'Department of Virology, College of Medicine, University of Ibadan, Ibadan, Nigeria 